\title{
Simulating and Modeling the Signal Attenuation of Wireless Local Area Network for Indoor Positioning
}

\author{
Terence Lines \\ Centre for Advanced Spatial \\ Analysis \\ University College London \\ London, United Kingdom \\ terence.lines.18@ucl.ac.uk
}

\author{
Anahid Basiri \\ Centre for Advanced Spatial \\ Analysis \\ University College London \\ London, United Kingdom \\ a.basiri@ucl.ac.uk
}

\begin{abstract}
Location is a key filter for mobile services, including navigation or advertising. However, positioning and localization inside buildings and in indoor spaces, where users spend most of their time and where the signals of the most widely-used positioning system, i.e. Global Navigation Satellite Systems such as GPS (Global Positioning System), are not available, can be challenging. In this regard, Wireless Local Area Networks (WLAN), e.g. Wi-Fi, can be used for positioning purposes by using a WLAN-enabled device, e.g. a smartphone, to measure and match the Received Signal Strength (RSS) of a signal broadcast by an access point. The challenges of this approach are that accurate maps of RSS are required, and that measuring RSS can be affected by many factors, including the dynamics of the environment and the orientation and type of a device. This paper provides a path-loss model to produce RSS maps automatically from floor plans and introduces an agent-based simulation approach to investigate different positioning methods. This provides a pathway to reduce the time and effort associated with WLAN positioning research.
\end{abstract}

\section{CCS CONCEPTS}

- Networks $\rightarrow$ Wireless local area networks • Computing methodologies $\rightarrow$ Modelling methodologies

\section{KEYWORDS}

WLAN signals, Indoor positioning, Signal fingerprinting, Agentbased modeling

\footnotetext{
Permission to make digital or hard copies of all or part of this work for personal or classroom use is granted without fee provided that copies are not made or distributed for profit or commercial advantage and that copies bear this notice and the full citation on the first page. Copyrights for components of this work owned by others than ACM must be honored. Abstracting with credit is permitted. To copy otherwise, or republish, to post on servers or to redistribute to lists, requires prior specific permission and/or a fee. Request permissions from Permissions@acm.org.

GeoSim'19, November 5, 2019, Chicago, IL, USA

(C) 2019 Association for Computing Machinery. ACM ISBN 978-1-4503-6956-5/19/11 ...\$15.00

https://doi.org/10.1145/3356470.3365527
}

\section{ACM Reference format:}

Terence Lines and Anahid Basiri. 2019. Simulation and Modeling the Signal Attenuation of Wireless Local Area Network for Indoor Positioning. In Proceedings of the 2nd ACM SIGSPATIAL International Workshop on GeoSpatial Simulation (GeoSim'19), November 5, 2019, Chicago, IL, USA. ACM, New York, NY, USA, 7 pages. https://doi.org/10.1145/3356470.3365527

\section{INTRODUCTION}

Many everyday services and applications, such as navigation, location-based advertising, and even emergency services, rely on location. While GPS, and generally Global Navigation Satellite Systems (GNSS), are the most widely used positioning technologies for outdoor use, the GNSS signals are not available or reliable for indoor use [14]. This is particularly important because we spend most of our time inside buildings. Studies in the US show that an average person spends over $80 \%$ of their time indoors [15]. Therefore, it is important to have a low-cost or ideally free, widely available and accessible technology that can provide an accurate and reliable Indoor Positioning System (IPS). Among many currently existing technologies, most of which use signals not initially designed for positioning, e.g. WLAN and Bluetooth Low Energy (BLE), Radio-Frequency Identification (RFID), Mobile Communication Networks, WLAN seems the best indoor positioning technology for a variety of applications and services such as indoor navigation, or emergency services [5].

WLAN antennas are cheap and already embedded in the smartphones, and broadcasting beacons can be found in many buildings. This provides high coverage and low cost, which have been identified as two important factors for an IPS to be used by a majority of indoor applications and services [5]. WLAN-based positioning mainly uses the Received Signal Strength Indicator (RSSI). The RSSI is a measure of signal strength between a WLAN beacon or Access Point (AP), which broadcast the signals and provides wireless connectivity to a wider network, and the antenna embedded in a WLAN-enabled device. However, WLAN signals can be easily affected by building features, e.g. walls and furniture, interference due to 
other signals [6], and the dynamic nature of their environment, e.g. people moving around.

In order to convert the RSSI measurements to a location, it is normal to match the measured RSSI against a previously produced RSSI map. There are two main approaches to producing an RSSI map (A) modelling the signal propagation, and (B) fingerprinting [8]. Fingerprinting currently seems to be more accurate but requires measurement on-site, which can be time-consuming and costly. If signal propagation modelling can be improved to handle measurement noise and the effects of the environment, it could facilitate automatic RSSI map creation.

Understanding the effects of measurement noise and the environment on RSSI is highly empirical, requiring access to physical locations to undertake experiments. While some may share their fingerprinting data sets and position traces to help reproduce results and allow further research (e.g. MendozaSilva et al [19]), these do not directly help investigate fundamental variables of accuracy such as building layout, WLAN beacon locations, underlying environment dynamics, and measurement methodology.

In this regard, this paper introduces an agent-based simulation approach for an IPS based on signal propagation modelling.

Compared to other modelling approaches, Agent-Based Modelling (ABM) can have the potential to represent smallscale processes in a more detailed and specific manner, allowing researchers to gain precise information on how a system functions [18]. Another strength of ABM is its ability to represent space and the relative locations of individuals accurately and explicitly. This feature can be of particular importance in situations where the characteristics of a particular spatial behavior could have a significant influence on global outcomes [21]. In this investigation, local spatial structure is of fundamental importance to model outcomes, so this advantage of ABM is especially relevant.

One issue that has often been highlighted with respect to $\mathrm{ABM}$ research is the difficulty of verification and validation of a model and its results. ABMs typically require different approaches from other computational models [27] and while various authors have presented their own schemes for "verification" and "validation", there is not universal agreement on what the terms mean in the context of ABM. For model verification, for example, some focus on good programming practice [10], others on whether a model replicates large scale patterns observed in the real world [21], while others have proposed narrower mathematical procedures, involving the representation of models in alternative forms (e.g. [11]. Since this paper serves to introduce a model as a foundation for future work, verification and validation are considered only in the sense of how well certain model behaviors match the findings of empirical research. Further work could include a deeper approach to these questions, along with considerations of sensitivity [10], and robustness [9].
The IPS produces maps of signal strength automatically from floor plans. Several experiments evaluate the effect on accuracy of the positioning solution of both map error and noise during signal strength measurement. A better understanding of error correction methods allows for faster, more robust data collection, including by crowdsourcing, which can further improve signal propagation models in a virtuous circle.

The paper is structured as follows: section 2 explains the WLAN-based positioning techniques, map creation and the sources and the impacts of noises on RSSI. Then the simulation methodology is discussed in section 3. Finally, section 4 discusses the experiments and the results, followed by a conclusion.

\section{WLAN-BASED POSITIONING}

There are several methods to convert RSSI measurements to a location [23]. The most straight-forward are geometric techniques, such as trilateration, however these are not generally used as they are based upon signal propagation in free space and are poorly suited to non-Line of Sight (LoS) readings typical of an indoor environment [23]. Instead, the main approaches match signal data to an RSSI map through deterministic and probabilistic methods. Deterministic methods associate each map location with a single set of RSSI values and find the best fit to the measured RSSI through minimizing a cost function such as a Euclidean distance [23]. A typical deterministic algorithm is k-nearest neighbor [4]. On the other hand, probabilistic methods consider each location to have a range of RSS values due to noise in RSS measurement. These may be described as histograms based on the results of fingerprinting [29] but are more compactly described as parametric distributions, e.g. Gaussian [12]. Probabilistic methods naturally lead to extended Bayesian approaches which maximize the probability over a set of measurements over time, such as conditional random fields [28], Kalman filters [1] and particle filters [20], or Hidden Markov Models [24].

Fingerprinting is the most accurate and widely used approach for RSSI map creation[4]. Fingerprinting is based on measuring RSSI at known locations through site survey, with considerable time and effort [4]. This initial effort must be repeated to update the fingerprint as it will otherwise decrease in accuracy as the underlying environment changes. Approaches to lessen or eliminate the survey effort include improved mapping and localization algorithms which require less fingerprinting data [2], proposals to crowdsource the fingerprinting data [26], and improvements to signal propagation modelling [16]. Signal propagation models use simplified knowledge of how signal strength reduces with distance and presence of obstacles to estimate RSSI from some inputs, e.g. an internal plan of the building. 
RSSI measurements are highly dependent on the measurement methodology. An RSSI value may be attenuated by several sources of systematic noise in the measurement: fading due to multiple signal paths [29]; device-based e.g. the choice of device used and device orientation [25]; user-based e.g. user orientation and the way a device is held [22]; and dynamic changes to the environment such as the presence of other people [22]. In a research setting, collection of testing data can be controlled to ensure the methodology is similar to the underlying fingerprint, however, any widely used IPS must allow for user heterogeneity. This requires methods to correct for this noise in measured RSSI data before comparison to an RSSI map. Additionally, the map itself needs to be as independent of measurement noise as possible. For fingerprinting, this is often obtained through additional effort e.g. multiple measurements are taken in different orientations at each location to account for this source of noise.

\section{METHODOLOGY}

Agent-Based Modelling typically involves the creation of a computer simulation in which entities in a real-world environment and their behaviors and interactions are represented explicitly, in order to study key features of a system [10]. The utility of this technique for the representation of human systems is well-established $[3,7]$.

The Netlogo package was used to build a model of a user being located by an IPS. This is not a traditional agent-basedmodel - it has only a single agent, whose only action is to move randomly to create RSSI readings for an IPS - however Netlogo can readily implement this and has the ability to systematically test changes to inputs.

\subsection{Environment and Entities}

The environment of a building is represented by a twodimensional (2-D) floorplan of walls. In this instance, the $70 \mathrm{~m}$ by $100 \mathrm{~m}$ floor of a stylized shopping center. Three types of entities exist within the environment, however only the user is referred as an agent in this paper, albeit the other two are modeled as "turtles" in Netlogo. Beacons are a static entity with nothing more than a location. The user is the entity being localized and moves around the environment. The third type of entity is people, moving around the environment and potentially affecting the RSSI measurement. While the user is affected by interactions with them, people themselves are not affected by interactions, hence they are not referred to in this paper as agents. In this simple instance, movements of the user and other people are assumed to be random and unconstrained by walls or other entities. A typical configuration is shown in Figure 1.

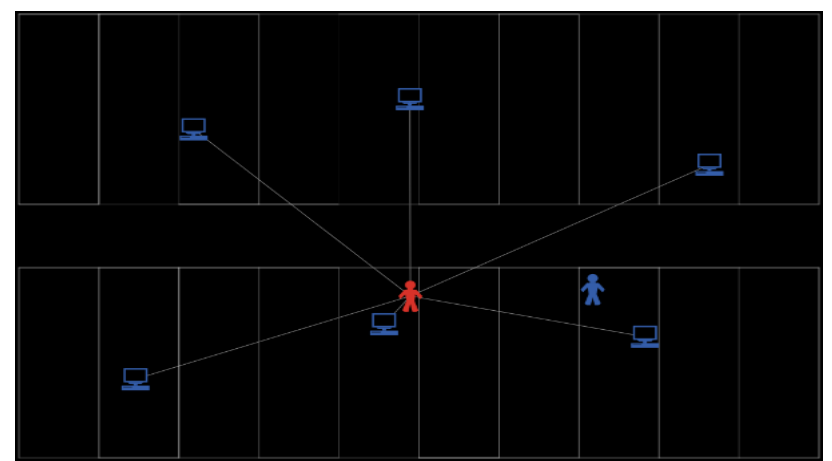

Figure 1: Simulation environment

\subsection{Map creation}

Creating synthetic RSSI values for the environment is achieved through signal propagation modelling. This will not be accurate to the real fingerprint for the reasons we are trying to improve signal propagation modelling. However, by producing a synthetic fingerprint with a detailed signal propagation model and then producing the map using a simplified signal propagation model with less floor plan information, it is believed that the spatially correlated errors are analogous to propagation modelling of real RSSI data, and that it is the relative error between RSSI value and the map which determines IPS accuracy.

Each $1 \mathrm{~m}^{2}$ cell has a synthetic fingerprint, generated by a standard signal propagation model known as the Wall Attenuation Factor (WAF) model [4] which takes the straightline distance between the user and a beacon along with the attenuation effect specific to any intervening walls. The model formula is:

$$
\mathrm{RSSI}=P L(1)-10 n \log _{10} d-\sum W A F
$$

Where PL(1) is RSSI at a distance of 1 meter, $d$ is distance in meters, $n$ represents the decrease in intensity with distance (depending on reflection and attenuation effects); and $W A F$ is the loss of signal due to attenuation through a wall.

Each cell also has a RSSI map approximation calculated with a simpler WAF model based on less information - not all walls are visible to the map model and the specific attenuation effect of each wall is unknown. 5 different map models were considered which provide a range of error against the fingerprint: the fingerprint itself can be used; two WAF models, one that has been fitted to the fingerprint data by linear regression and one with pre-determined parameters (based on the average wall attenuation); and two free space models which do not account for the presence of walls, again with one fitted to the data and one with pre-determined parameters. The simulation parameters controlling wall attenuation and visibility were modified so the fitted WAF had a typical Root Mean Square Error (RMSE) against the fingerprint of circa 3-5 $\mathrm{dBm}$, reflecting empirical results [4]. 


\subsection{Positioning}

To estimate their position, the user generates an RSSI scan for their current position, generated by the fingerprint plus noise during measurement, and compares it against the map using an algorithm. The IPS accuracy is measured as the distance between the estimate and actual location.

There are 4 types of measurement noise considered in the model: fading, attenuation due to the movement of people across the signal paths, device heterogeneity and orientation. Parameters for each were chosen as follows: fading noise is represented as a Gaussian distribution with standard deviation $2.5 \mathrm{dBm}$ to give a typical range of $10 \mathrm{dBm}$ [29]; each person in the direct signal path attenuated the signal $5 \mathrm{dBm}$, scaling down a $10 \mathrm{dBm}$ effect noted in LoS situations [22]; device heterogeneity was represented by a change in RSSI measurements between model runs, uniformly distributed between $-10 \mathrm{dBm}$ and $10 \mathrm{dBm}$ [25]; and orientation was represented by a bell-shaped attenuation with peak of $15 \mathrm{dBm}$ and width of 1.2 radians.

Two well-known positioning algorithms were used: a knearest-neighbor (k-NN) algorithm with $\mathrm{k}=3$; and a Bayesian algorithm based on each cell having a Gaussian distribution with mean equal to the fingerprint and identical standard deviation.

\subsection{Experiments}

Two experiments are undertaken to analyze the effect on location error:

- Investigating variance in the number of RSSI readings and algorithm, through changing the number of beacons and taking multiple scans for each position estimate (within a single second so with the user at the same location). The scenarios and observations are summarized in Table 1. For each of the 18 scenarios of interest, 80 permutations of path-loss and noise error were generated, with each permutation repeated five times, for a total of 7,200 different model runs. Each model was run for 300 steps, with average errors measured across all steps.

- Investigating the interaction of the errors in the path loss model and error due to noise effects. Beacon numbers and scans per estimate were held constant at moderate values. The k-NN algorithm was used as it had a shorter running time. The scenarios and observations are summarized in Table 2. There were 80 permutations of path-loss and noise error, as before, with each permutation repeated ten times for a total of 800 model runs. Each model was run for 300 steps, with average errors measured across all steps.
Table 1: Setup of experiment 1: variance in number of RSSI readings

\begin{tabular}{|c|c|}
\hline Parameter & Variation \\
\hline Number of beacons & $3,6,12$ \\
\hline Scans per estimate & $1,4,8$ \\
\hline Algorithms & k-NN, Bayesian \\
\hline Path loss model & $\begin{array}{l}\text { Fingerprint, WAF, unfitted WAF, } \\
\text { free space, unfitted free-space }\end{array}$ \\
\hline Noise effects & $\begin{array}{l}\text { Device heterogeneity on / off } \\
\text { Device directionality on / off } \\
\text { Number of people } 0246\end{array}$ \\
\hline Observation & Description \\
\hline Location error & $\begin{array}{l}\text { Mean average location error } \\
\text { (metres) }\end{array}$ \\
\hline RSSI error & $\begin{array}{l}\text { RMSE of error between expected } \\
\text { RSSI at user's location and the } \\
\text { actual RSSI caused by path loss } \\
\text { errors and noise effects (dBm) }\end{array}$ \\
\hline
\end{tabular}

Table 2: Setup of experiment 2: interaction of location and noise error

\begin{tabular}{|c|c|}
\hline Parameter & Variation \\
\hline Number of beacons & 6 \\
\hline Scans per estimate & 4 \\
\hline Algorithms & $\mathrm{k}-\mathrm{NN}$ \\
\hline Path loss model & $\begin{array}{l}\text { Fingerprint, WAF, unfitted WAF, } \\
\text { free space, unfitted free-space }\end{array}$ \\
\hline Noise effects & $\begin{array}{c}\text { Device heterogeneity on / off } \\
\text { Device directionality on / off } \\
\text { Number of people } 0246\end{array}$ \\
\hline Observation & Description \\
\hline Location error & $\begin{array}{c}\text { Mean average location error } \\
\text { (metres) }\end{array}$ \\
\hline Path-loss error & $\begin{array}{l}\text { RMSE of error between expected } \\
\text { RSSI at user's location and the } \\
\text { actual RSSI caused by path loss } \\
\text { error (dBm) }\end{array}$ \\
\hline Noise error & $\begin{array}{l}\text { RMSE of error caused by noise } \\
\text { effects }(\mathrm{dBm})\end{array}$ \\
\hline
\end{tabular}

\section{RESULTS}

For a constant number of beacons, increasing the number of scans decreased location error for a given level of RSSI error. The improvement is only material for low overall levels of RSSI error, in the order of $5 \mathrm{dBm}$ and below. This is as expected, as multiple scans at the same location generates multiple measurements of fading error but other errors are identical. 
Hence, when fading error is a small contribution to overall error, additional scans do not improve accuracy.

Increasing the number of beacons decreased the location error at all values of RSSI error. It is believed that this effect is due to having beacons closer to the user. This improves positioning as closer beacons have greater signal change between adjacent cells.

The K-NN and Bayesian algorithms performed similarly. This is an artefact of the modelling process, as without knowledge of the RSSI variance at each location, maximizing the Gaussian probability is effectively identical to minimizing the Euclidean distance between signals, as per the k-NN algorithm.

At high levels of RSSI error, the location error appeared to have an upper bound of around $45 \mathrm{~m}$, based on the relative performance of estimating position randomly. This upper bound has no significance beyond the model environment size and modelling of user movement. For all categorizations of results there was a breakpoint around $5 \mathrm{dBm}$ RSSI where the gradient of location error against RSSI error decreased.

The combined effects of path-loss error and noise error (as calculated through an IDW interpolation) are shown in Figure 2. Location error increases most quickly as path loss and noise errors increase to $5 \mathrm{dBm}$. The change in location error is less pronounced thereafter, which confirms the results shown in Figure 3. Both error types have a similar effect on location error, except that the worst-performing path-loss models (i.e. the unfitted free-space model) have an exceptionally high location error, which is likely due to its bias to over-estimating distances.

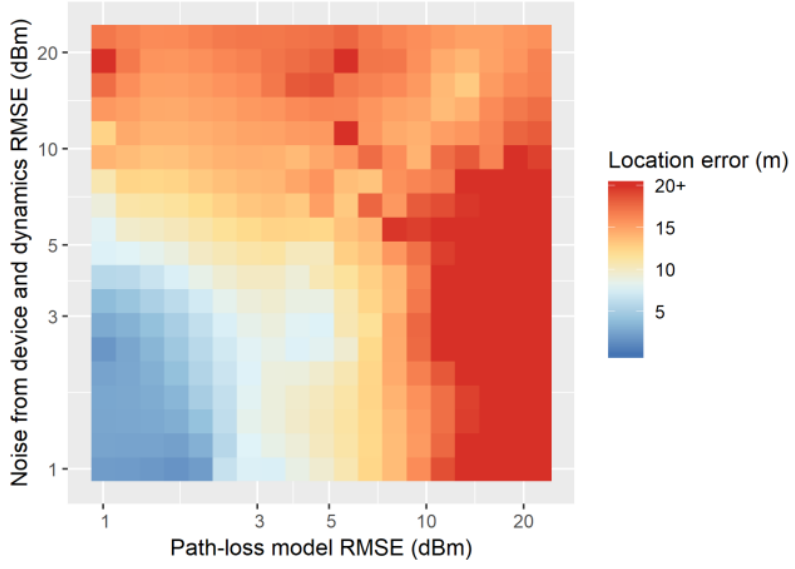

Figure 2: Effect of noise and path-loss errors on location error

An interesting effect of the interaction between the two error types can be seen: location errors form two peaks with high path-loss error and low noise error, and vice versa. If error levels are already high, an increase in the other error type has little effect.

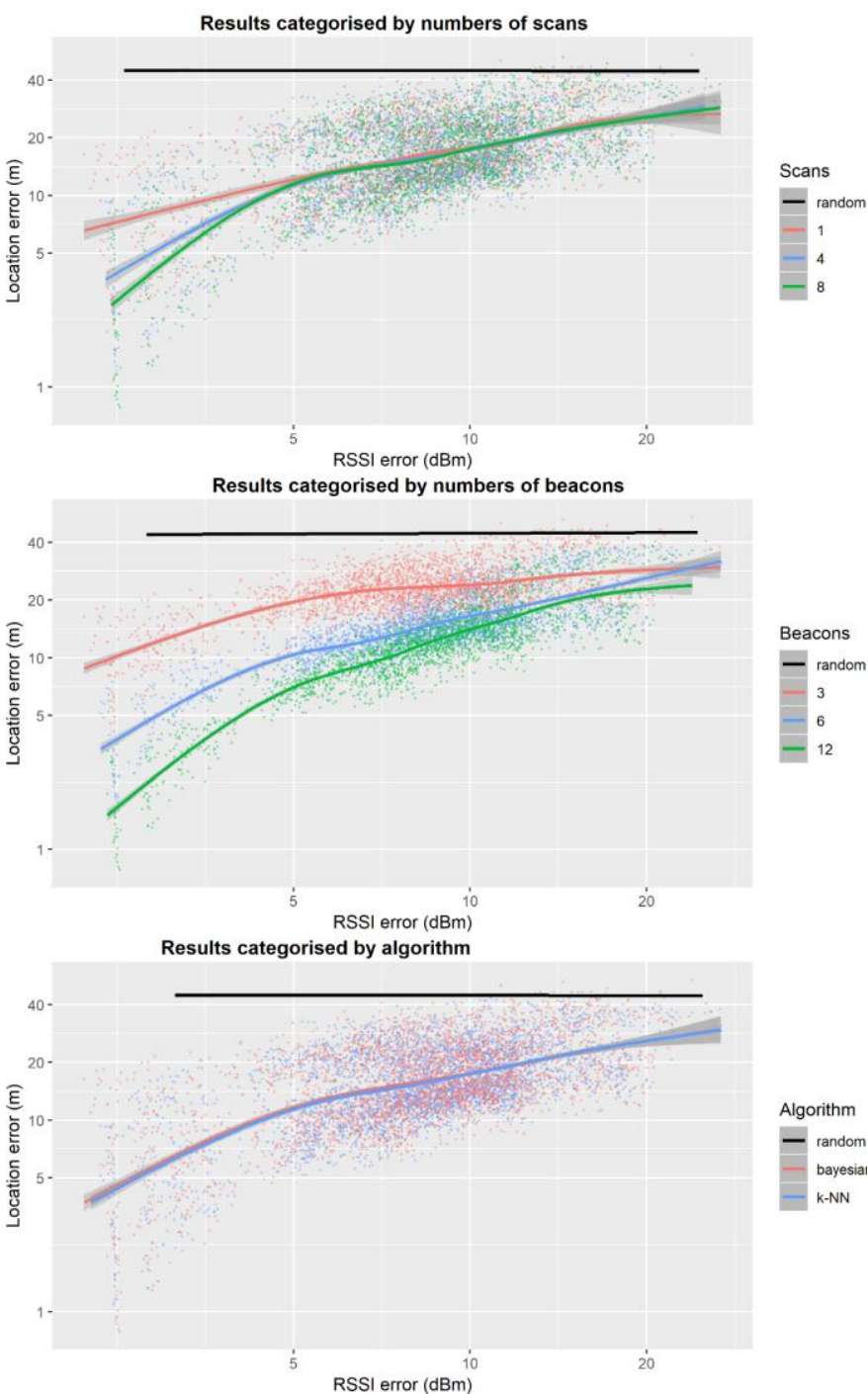

Figure 3: Results for varying measurements and algorithm

\section{CONCLUSION AND FUTURE WORK}

This paper demonstrates the capability of a simulation to evaluate different aspects of indoor positioning services performance. Specifically, it reproduces expected phenomena of greater accuracy with increased numbers of scans and beacons and it explores the relationship of IPS accuracy against two underlying types of error, RSSI map quality and measurement noise. It identifies a rapid decrease in accuracy within the first $5 \mathrm{dBm}$ of error, highlighting the difficulty of creating a system that works robustly in real-world conditions. The proposed system can work with a wide range of sources of error: the ability to consider different RSSI map quality, the inclusion of noise from other people, the possibility of heterogeneous devices and a sophisticated model of device attenuation. 
Using this framework to replicate existing empirical research fulfils a dual purpose of validating the model and generalizing empirical results from their specific environments and methodologies.

Future work will hopefully include investigating the robustness and convergence conditions for recently proposed error correction methods based on crowdsourcing and unsupervised machine learning. This would require multiple agents with more sophisticated agent behavior e.g. movement. Also, comparing the results of models with real-world RSS data can give a better understanding of the replicability of the model. This will also help to recognize other potential sources of errors to be incorporated in an ultimately universal model. Such model needs a robust measure that can quantify the effects of the incorporated parameters. For this a variancebased global sensitivity analysis $[13,17]$ can be carried out.

\section{ACKNOWLEDGMENTS}

Authors appreciate the support from UK Research and Innovation (UKRI). This work presented in this paper has been partially funded by the UK Research and Innovation (UKRI) Future Leaders Fellowship (grant number: MR/S01795X/1) for University College London (UCL).

Authors would like to thank Dr Thomas Evans who generously contributed to the paper, in particular on the justification of using ABM for the purpose of indoor positioning.

\section{REFERENCES}

[1] S. Ali-Loytty, T. Perala, V. Honkavirta, and R. Piche. 2009. Fingerprint Kalman Filter in indoor positioning applications. In 2009 IEEE Control Applications, (CCA) Intelligent Control, (ISIC), 1678-1683. DOI:https://doi.org/10.1109/CCA.2009.5281069

[2] M.S. Anwar, F. Hossain, N. Mehajabin, M. Mamun-Or-Rashid, and M.A. Razzaque. 2018. A Comparative Study on Gaussian Process Regressionbased Indoor Positioning Systems. In 2018 International Conference on Innovation in Engineering and Technology (ICIET), 1-5. DOI:https://doi.org/10.1109/CIET.2018.8660860

[3] R. Axelrod. 1997. Advancing the art of simulation in the social sciences. In Simulating social phenomena. Springer, 21-40.

[4] P. Bahl and V.N. Padmanabhan. 2000. RADAR: an in-building RF-based user location and tracking system. In Proceedings IEEE INFOCOM 2000. Conference on Computer Communications. Nineteenth Annual Joint Conference of the IEEE Computer and Communications Societies (Cat. No.00CH37064), 775-784 vol.2. DOI:https://doi.org/10.1109/INFCOM.2000.832252

[5] A. Basiri, E.S. Lohan, T. Moore, A. Winstanley, P. Peltola, C. Hill, P. Amirian and P. Figueiredo e Silva. 2017. Indoor location based services challenges, requirements and usability of current solutions. Computer Science Review 24, (May 2017), 1-12. DOI:https://doi.org/10.1016/j.cosrev.2017.03.002

[6] A. Behboodi, N. Wirstrom, F. Lemic, T. Voigt, and A. Wolisz. 2015 Interference Effect on Localization Solutions: Signal Feature Perspective. In 2015 IEEE 81st Vehicular Technology Conference (VTC Spring), 1-7. DOI:https://doi.org/10.1109/VTCSpring.2015.7145885

[7] E. Bonabeau. 2002. Agent-based modeling: Methods and techniques for simulating human systems. Proceedings of the national academy of sciences 99, suppl 3 (2002), 7280-7287.

[8] P. Davidson and R. Piché. 2017. A Survey of Selected Indoor Positioning Methods for Smartphones. IEEE Communications Surveys Tutorials 19, 2 (Secondquarter 2017), 1347-1370. DOI:https://doi.org/10.1109/COMST.2016.2637663

[9] P.K. Dunstan and C.R. Johnson. 2005. Predicting global dynamics from local interactions: individual-based models predict complex features of marine epibenthic communities. Ecological Modelling 186, 2 (2005), 221-233.
[10] N. Gilbert. 2008. Agent-based models. Sage.

[11] P. Gómez-Mourelo. 2005. From individual-based models to partial differential equations: An application to the upstream movement of elvers. Ecological modelling 188, 1 (2005), 93-111.

[12] A. Haeberlen, E. Flannery, A.M. Ladd, A. Rudys, D.S. Wallach, and L.E Kavraki. 2004. Practical robust localization over large-scale 802.11 wireless networks. In Proceedings of the 10th annual international conference on Mobile computing and networking - MobiCom '04, 70. DOI:https://doi.org/10.1145/1023720.1023728

[13] J.Y. Kang and J. Aldstadt. 2019. Using multiple scale space-time patterns in variance-based global sensitivity analysis for spatially explicit agentbased models. Computers, Environment and Urban Systems 75, (2019), 170-183.

[14] M.B. Kjærgaard, H. Blunck, T. Godsk, T. Toftkjær, D.L. Christensen, and K. Grønbæk. 2010. Indoor positioning using GPS revisited. Lecture Notes in Computer Science (including subseries Lecture Notes in Artificial Intelligence and Lecture Notes in Bioinformatics) 6030 LNCS, (2010), 3856. DOI:https://doi.org/10.1007/978-3-642-12654-3_3

[15] N.E. Klepeis, W.C. Nelson, W.R. Ott, J.P. Robinson, A.M. Tsang, P. Switzer, J.V. Behar, S.C. Hern, and W.H. Engelmann. 2001. The National Human Activity Pattern Survey (NHAPS): a resource for assessing exposure to environmental pollutants. Journal of Exposure Science \& Environmental

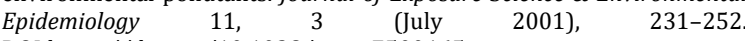
DOI:https://doi.org/10.1038/sj.jea.7500165

[16] J. Kwon, B. Dundar, and P. Varaiya. 2004. Hybrid algorithm for indoor positioning using wireless LAN. In IEEE 60th Vehicular Technology Conference, 2004. VTC2004-Fall. 2004, 4625-4629 Vol. 7 DOI:https://doi.org/10.1109/VETECF.2004.1404967

[17] A. Ligmann-Zielinska. 2018. 'Can You Fix It?'Using Variance-Based Sensitivity Analysis to Reduce the Input Space of an Agent-Based Model of Land Use Change. In GeoComputational Analysis and Modeling of Regional Systems. Springer, 77-99.

[18] C.M. Macal. 2010. To agent-based simulation from system dynamics. In proceedings of the winter simulation conference, 371-382.

[19] G. Mendoza-Silva, P. Richter, J. Torres-Sospedra, E. Lohan, and J. Huerta. 2018. Long-term WiFi fingerprinting dataset for research on robust indoor positioning. Data 3, 1 (2018), 3.

[20] A. Rai, K.K. Chintalapudi, V.N. Padmanabhan, and R. Sen. 2012. Zee: zeroeffort crowdsourcing for indoor localization. In Proceedings of the 18th annual international conference on Mobile computing and networking Mobicom '12, 293. DOI:https://doi.org/10.1145/2348543.2348580

[21] S.F. Railsback and V. Grimm. 2019. Agent-based and individual-based modeling: a practical introduction. Princeton university press.

[22] F.D. Rosa, M. Pelosi, and J. Nurmi. 2012. Human-Induced Effects on RSS Ranging Measurements for Cooperative Positioning. International Journal of Navigation and Observation 2012, (2012), 1-13. DOI:https://doi.org/10.1155/2012/959140

[23] F. Seco, A.R. Jimenez, C. Prieto, J. Roa, and K. Koutsou. 2009. A survey of mathematical methods for indoor localization. In 2009 IEEE International Symposium on Intelligent Signal Processing, 9-14. DOI:https://doi.org/10.1109/WISP.2009.5286582

[24] J. Seitz, J. Jahn, J.G. Boronat, T. Vaupel, S. Meyer, and J. Thielecke. 2010. A Hidden Markov Model for urban navigation based on fingerprinting and pedestrian dead reckoning. In 2010 13th International Conference on Information Fusion,

DOI:https://doi.org/10.1109/ICIF.2010.5712025

[25] B. Viel and M. Asplund. 2014. Why is fingerprint-based indoor localization still so hard? In 2014 IEEE International Conference on Pervasive Computing and Communication Workshops (PERCOM WORKSHOPS), 443-448.

[26] H. Wang, S. Sen, A. Elgohary, M. Farid, M. Youssef, and R.R. Choudhury. 2012. No need to war-drive: unsupervised indoor localization. In Proceedings of the 10th international conference on Mobile systems,

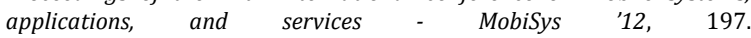
DOI:https://doi.org/10.1145/2307636.2307655

[27] X. Xiang, R. Kennedy, G. Madey, and S. Cabaniss. 2005. Verification and validation of agent-based scientific simulation models. In Agent-directed simulation conference, 55.

[28] Z. Xiao, H. Wen, A. Markham, and N. Trigoni. 2014. Lightweight map matching for indoor localisation using conditional random fields. In IPSN-14 Proceedings of the 13th International Symposium on Information Processing in Sensor Networks, 131-142. DOI:https://doi.org/10.1109/IPSN.2014.6846747

[29] M. Youssef and A. Agrawala. 2005. The Horus WLAN location determination system. In Proceedings of the 3rd international conference on Mobile systems, applications, and services - MobiSys '05, 205. DOI:https://doi.org/10.1145/1067170.1067193 
